Revue d'histoire de l'Amérique française

REVUE D.HISTOIRE DE L'AMÉRIQUE FRANÇAISE

\title{
Quelques documents nouveaux sur Nicolas Denys
}

\section{René Baudry}

Volume 9, numéro 1, juin 1955

URI : https://id.erudit.org/iderudit/301691ar

DOI : https://doi.org/10.7202/301691ar

Aller au sommaire du numéro

Éditeur(s)

Institut d'histoire de l'Amérique française

ISSN

0035-2357 (imprimé)

1492-1383 (numérique)

Découvrir la revue

Citer cet article

Baudry, R. (1955). Quelques documents nouveaux sur Nicolas Denys. Revue d'histoire de l'Amérique française, 9(1), 14-30. https://doi.org/10.7202/301691ar d'utilisation que vous pouvez consulter en ligne.

https://apropos.erudit.org/fr/usagers/politique-dutilisation/ 


\section{QUELQUES DOCUMENTS NOUVEAUX SUR NICOLAS DENYS}

On connaît très peu d'autographes de Nicolas Denys. Le professeur Ganong, dans la magistrale édition de ses ouvrages publiée par la Société Champlain, ${ }^{1}$ a reproduit la seule signature alors connue de lui. Les archivistes de La Rochelle ont, depuis ce temps, trouvé d'autres pièces signées de son nom, et le Rév. W. Inglis Morse ${ }^{2}$ a publié une lettre originale. Au total, si nous ajoutons quelques copies de lettres perdues, cela représente à peine deux cents lignes d'écriture. Cette rareté pouvait laisser croire que Denys, à part ses livres, probablement revus par un ami, n'avait presque rien écrit, et confirmer la réputation d'illettré que l'historien Moreau a voulu lui attribuer.

Heureusement pour nous, les archives de Rennes ont conservé une liasse de documents qui nous apporte à ce sujet une bonne surprise. Un dossier, découvert en 1914 par M. Bourde de la Rogerie, ${ }^{3}$ puis perdu et retrouvé, relate l'histoire d'une compagnie de commerce formée entre Nicolas Denys, Cristophe Fouquet de Chalain, cousin du surintendant Fouquet, et les deux frères Jacob et Abraham Duquesne. Cette documentation provient des papiers de Christophe Fouquet, et le titre

1 W. F. Ganong (éd.). Denys Description and Natural History of Acadia (Toronto, The Champlain Society, 1908). Les nouveaux documents de Rennes et de La Rochelle permettent de rectifier quelques erreurs et de combler quelques lacunes de cet ouvrage. Mais il demeure, par sa bibliographie et ses notes érudites, la source principale de renseignements sur Nicolas Denys.

2 Gravestones of Acadia. London, Quaritch, 1929.

$3 \mathrm{M}$. Henri Bourde de la Rogerie archiviste aux Archives départementales d'Ille-et-Vilaine, avait remarqué cette liasse (2E, Sup. 248) et lui consacra une communication au Congrès des Sociétés savantes. Il projetait d'écrire un livre sur Nicolas Denys, mais n'en rédigea que quelques chapitres restés inédits. 
indique qu'elle servit à un avocat, dans des poursuites judiciaires contre Denys. Elle contient 105 pièces, parmi lesquelles on trouve des lettres originales de Jacob et Abraham Duquesne, quelques lettres d'Emmanuel LeBorgne, et surtout 23 lettres et mémoires de la main de Nicolas Denys, dont plusieurs datés de Percé, Saint-Pierre et Chedabouctou.

Cette correspondance nous fournit beaucoup d'indications nouvelles pour la biographie de Denys et pour l'histoire de l'Acadie. Ses deux volumes nous ont sans doute appris l'essentiel de sa carrière, mais ces lettres les complètent et les éclairent. Il nous dit par exemple qu'il était « de famille d'ingénieurs». Il nous fournit des renseignements sur ses concurrents, le marchand rochelais LeBorgne et les deux associés DuCanger et LaGiraudière, si peu connus que Ganong les a crus un seul personnage. Il nous apprend surtout l'existence de cette association avec Cristophe Fouquet et les Duquesne, dont il ne souffle pas un seul mot dans ses ouvrages. Deux bonnes raisons, croyons-nous, expliquent ce silence: c'est qu'au moment où il écrivait, il leur devait des sommes considérables et ne désirait pas attirer l'attention là-dessus; puis, dédiant son livre au Roi, il préférait ne pas étaler ses relations avec deux personnages compromettants, l'un cousin de l'ex-surintendant disgracié, l'autre un huguenot irréductible, à qui on ne pardonnait son attachement au protestantisme que parce qu'il était nécessaire à la France.

Ces lettres nous montrent en détail le fonctionnement d'une compagnie de commerce: son lancement, quelques années de succès partiels, de vigoureux efforts pour la renflouer, puis son écroulement sous l'avalanche des forces contraires. En lisant, sous la plume de Denys, le journal de ses difficultés, on comprend vraiment pourquoi lui-même et la plupart de ses imitateurs échouèrent. Son aventure présente une valeur typique, dont on peut tirer des conclusions générales. Sans vouloir réécrire la biographie de Denys, nous voudrions seulement, en feuilletant le dossier de Rennes, étudier cette phase de sa vie (1654-1664) qui correspond à cette association. 
Au moment où Nicolas Denys se lance dans cette nouvelle entreprise, il est déjà un vieil habitué des côtes acadiennes. Depuis son arrivée au pays avec Razilly en 1633, il a résidé plusieurs années à Port Rossignol, La Hève et Miscou, et organisé de nombreux voyages de pêche ou de traite sur les côtes de Terre-Neuve et d'Acadie. Mais la déveine, jusque là, s'est acharnée contre lui: les Portugais ont pris un de ses vaisseaux, d'Aulnay lui a enlevé les forts de Miscou et Nepisiguit, LeBorgne a pillé Saint-Pierre. Rendu à l'âge de cinquante ans, il est riche, à défaut d'argent, d'une longue expérience. Malgré quelques dettes, son crédit demeure bon et il possède beaucoup de relations dans les ports. Il vient d'obtenir une nouvelle concession de la Compagnie de la Nouvelle-France, et des lettres-patentes du Roi l'établissent lieutenant-gouverneur. Bref, il est parvenu à ce moment de l'existence où un brasseur d'affaires malchanceux croit avoir atteint le terme de ses déboires et espère enfin forcer le succès. Un capitaine de navire, qu'il connait probablement de vieille date, Jacob Duquesne, lui avance à Nantes, en 1654, la somme de 4,500 L. et le met en relations avec son frère. Lions plus ample connaissance avec ces personnages.

La famille Duquesne ${ }^{4}$ entretenait depuis longtemps des rapports avec le Canada. Le père, Abraham (trois générations de Duquesne porteront ce prénom judaïque) à la fois marin et armateur, résidait à Dieppe. Avec un autre marchand huguenot, Dujardin, il avait financé un voyage de Poutrincourt en 1610 , et s'opposa catégoriquement à l'embarquement des pères Jésuites Biard et Massé. ${ }^{5}$ En 1629, il détenait une charte-partie avec le capitaine Daniel, ${ }^{6}$ quand celui-ci vint déloger Lord Ochiltree du Cap-Breton. Ses trois fils, Etienne, Abraham et Jacob exercèrent comme lui le métier de marins, d'armateurs, et, à l'occasion, de corsaires. Comme Mazarin négligeait la marine, les officiers trouvaient le loisir de commercer et d'organiser des campagnes à leur compte. Les trois frères Du-

${ }^{4}$ Le Rév. Père L. LeJeune, dans son Dictionnaire ..., a un peu confondu les membres de cette famille. Voir A. Jal, Abraham Duquesne et la marine de son temps, (2 vol., Paris, Plon, 1873).

5 G. Marcel, Factum ... de Poutrincourt (Paris, Maisonneuve, 1887), 9.

6 B. N., N.A.F. 4967 : 68. 
quesne prirent du service en Suède. En 1657, on voit avec inquiétude Etienne et Jacob, à la tête d'un équipage espagnol, et montés sur un navire du Roi, s'emparer d'un terreneuvier français. ${ }^{7}$ Abraham II, du gain de ses courses, avait acheté un magnifique domaine sur la rivière de Moros, ${ }^{8}$ à un kilomètre en amont de Concarneau. Il acquit aussi les terres des alentours, édifia des entrepôts et fit aménager sur sa propriété un quai où les vaisseaux de faible tonnage pouvaient aborder de toutes marées. De sa maison, bâtie sur une colline, il pouvait contempler le port de Concarneau et l'océan illimité. Aujourd'hui une statue de Duquesne, érigée à l'entrée de l'avenue, rappelle encore son souvenir. Les arbres du parc cachent la mer, mais les édifices ont gardé grande allure. Son jeune frère Jacob demeurait alors avec lui, et tous deux collaboraient étroitement dans toutes leurs entreprises. Grâce à eux, Denys connut un autre personnage influent de la région, Christophe Fouquet de Chalain.

La famille Fouquet ${ }^{9}$ s'intéressait aussi depuis longtemps au Canada. L'aïeul, François, marchand d'Angers, avait donné à ses deux fils une excellente éducation. L'aîné devint conseiller au Parlement de Paris et entra au Conseil du Roi. Richelieu l'avait chargé des affaires maritimes. Il fit partie de la Compagnie des Cent-Associés, dont les membres se réunissaient chez lui. Il possédait des terres à Beaupré, que sa veuve céda plus tard aux Hospitalières de Québec. ${ }^{10}$

Le cadet émigra en Bretagne et devint conseiller au Parlement de Rennes. Les enfants de ces deux frères réussirent également bien. A Paris, l'un devint évêque et l'autre, Nicolas, s'illustra comme Surintendant des finances. A Rennes, leur cousin, Christophe Fouquet de Chalain ${ }^{11}$ succédait à son père

7 Factum pour Julien Artur, Robert le Blanc et al... contre Jacob Duquesne, etc. B.N. Ff. 18591, f. 44-47.

${ }^{8} \mathrm{~L}$. Véron, Duquesne aux Moros (Paris, Plon, 1894), $36 \mathrm{p}$.

$9 \mathrm{~J}$. Lair, Nicolas Fouquet, procureur-général, surintendant des finances, ministre d'état sous Louis XIV, (2 vol., Paris, Plon, 1890).

${ }^{10} \mathrm{BRH}$, XXVII (1921) : 349 .

11 Pour la biographie de Fouquet de Chalain, nous avons consulté deux études inédites de M. Bourde de la Rogerie, dont un condensé paraîtra prochainement dans le Bulletin de la Soc. d'Hist. et d'Archéol. de Bretagne. 
dans la charge de conseiller. Il devint ensuite procureur général et président au Parlement de Bretagne. Ses fonctions n'empêchaient pas ce grave magistrat de commercer. Il possédait plusieurs vaisseaux, le Saint-Jean, le Petit Chalain et le Grand Chalain, et cumulait les charges de Capitaine et de Gouverneur de Concarneau. Il semble avoir exercé une influence magique sur la sénéchaussée de cette petite ville, dont les officiers savaient habilement concilier la justice avec les intérêts du Président et de ses amis. Ces Fouquet, comme tous les fonctionnaires de l'ancien régime issus de la bourgeoisie, les Colbert et les Bégon, entretenaient un esprit de famille très développé et s'entr'aidaient volontiers. Christophe Fouquet de Chalain et son cousin le Surintendant conclurent ensemble plusieurs marchés. Quand Nicolas Fouquet mit sur pied ses projets d'entreprises coloniales, il acheta de son cousin le port de Concarneau et des navires, qu'il ne lui paya d'ailleurs jamais.

Ces deux familles, les Duquesne et les Fouquet, nous fournissent un curieux exemple d'hérédité coloniale. De père en fils, elles transmettent à leurs descendants le goût du commerce, la passion de la mer et l'intérêt pour le Canada. Cette influence agit même à plusieurs générations de distance, puisqu'un siècle plus tard, un petit-fils d'Etienne Duquesne, Ange DuquesneMenneville, deviendra gouverneur du Canada.

Voilà donc les quatre partenaires qui entrent en relations vers 1654, pour exploiter la concession et les privilèges de Denys. Ce dernier propose d'abord à ses amis de former une puissante société de 20 à 25 personnes fournissant chacune au moins 4,000 L., pour la pêche à Terre-Neuve. Peut-être le surintendant Fouquet, déjà intéressé dans la Compagnie des Baleines, avait-il dès lors des visées sur le territoire de TerreNeuve, ${ }^{12}$ et son cousin dissuada-t-il Denys de toute entreprise de ce côté ? De toutes façons ce projet de grande envergure dut effrayer les prêteurs et ne reçut pas de suite. Le président de Chalain commença prudemment en 1655, par une simple

12 G. Marcel, Le surintendant Fouquet, vice-roi d'Amérique. Extrait de la Revrie de Géographie (Paris, 1885), $16 \mathrm{p}$. 
avance à Jacob Duquesne, pour un voyage au Canada. Chacun d'eux fournit 10,500 L. Le nom de Denys ne figure pas à l'acte, mais il dirigeait l'expédition.

L'année suivante, un contrat beaucoup plus explicite résume les accords antérieurs et nous expose les conditions détaillées d'une association en règle entre Nicolas Denys, Jacob Duquesne et Fouquet de Chalain. Pour le voyage de cette année et les précédents, Duquesne et Chalain ont prêté $40,220 \mathrm{~L}$. Denys s'engage à ramener le navire à Concarneau et à remettre pelleteries et poisson à ses associés, pour la vente. Si le revenu de l'année ne suffit pas à payer les dépenses, Denys payera 11/2\% d'intérêt par mois, soit $15 \%$ d'intérêt annuel. Il laisse en garantie l'original de sa concession à Jacob Duquesne.

Ce contrat appelle quelques remarques. L'intérêt de $15 \%$ paraîtrait aujourd'hui fort exagéré. A l'époque, on trouve souvent, dans des contrats de ce genre, des taux semblables et même supérieurs. ${ }^{13}$ Dans les prêts dits "à la grosse aventure », le taux variait entre 20 et $35 \%$, selon les risques encourus. Dans les expéditions de course et en temps de guerre, il atteignait même $50 \%$. Mais dans le cas présent, il faut noter que Denys paye les matelots et les engagés, et assume tous les risques, commerciaux et maritimes. Les prêteurs touchent un revenu fixe de $15 \%$ sur leur mise, sans compter de menus bénéfices accessoires. Il semble donc que Denys acceptait des conditions plutôt onéreuses. Il ne tarda pas d'ailleurs à protester contre ce taux d'intérêt qui en peu d'années doublait le capital.

Le nom D'Abraham Duquesne ne figure pas à l'acte d'association. Mais l'ensemble des circonstances montre avec évidence qu'il avait partie liée avec son frère. On le voit s'occuper de l'armement des vaisseaux; il envoie des rapports à Chalain; on le consulte sur les préparatifs de nouveaux embarquements. Après la mort de son frère, il ne cesse de harceler Denys, non seulement comme tuteur de son neveu, mais bien en son nom personnel.

${ }^{13} R H A F$, vol. IV, No 4 (mars 1951): 469-511. Marcel Delafosse, « La Rochelle et le Canada au XVIIIe siècle 》. 
Cette association allait diriger les activités de Denys pendant une dizaine d'années approximativement, de 1654 à 1664. Les cinq premières années, les associés organisent des embarquements annuels. Le navire part au printemps, au début de mai, fait la pêche, et revient en octobre avec une cargaison de morues et de fourrures. Quelques hommes demeurent à l'habitation pour la traite d'hiver. Denys utilise d'abord un navire à lui, puis pendant trois ans le Petit Saint-Jean, appartenant à Fouquet de Chalain, et la dernière année probablement un navire confisqué.

Un mémoire de 1657 nous renseigne sur la nature de ces embarquements. Le personnel compte une quinzaine de matelots, 10 soldats et 16 hommes de métier, en tout 42 hommes. Les marchandises comprennent des matériaux de construction, clous et ferrements; des vivres pour la traversée, biscuit, lard et pois; des provisions pour l'habitation, poudre à canon, farine, vins; des articles pour la traite, petites et grandes haches de Bayonne, poudre à fusil. La quantité de boissons emportées, 19 tonneaux de vin rouge et blanc, 12 barriques d'eau-de-vie, 5 tonneaux de cidre et 10 tonneaux de bière, montre que ces hommes avaient de bonnes soifs. Chaque embarquement coûtait de 12,000 à $15,000 \mathrm{~L}$.

On se rend aisément compte que de semblables expéditions, avec en plus les salaires de 40 employés, occasionnaient des dépenses considérables. Aussi le revenu ne permit-il, chaque année, que de rembourser une partie des avances, et les déficits s'accumulèrent. Si bien qu'en 1658, lors d'un arrêté de comptes, Nicolas Denys se trouvait débiteur d'une somme de 51,520 L. A l'automne, il envoie en France un navire chargé de poisson, mais lui-même, sans doute pour éviter des explications pénibles, demeure au Canada. Il avait d'ailleurs l'intention de s'y établir à demeure, puisqu'il y avait amené sa famille.

A partir de cette année, afin de limiter les dépenses, il n'organise plus d'armement autonome, mais fait venir vivres et marchandises par des vaisseaux normands, et leur remet en échange ses fourrures. Ce système gêne ses affaires d'une autre façon, car il doit payer $50 \%$ de la valeur des marchandises 
pour leur transport. Aussi n'envoie-t-il plus rien à ses créanciers les années suivantes. Duquesne et Fouquet s'étonnent de ne rien recevoir; Denys tâche de leur faire prendre patience et s'applique à l'économie. En 1660, il supprime les soldats et déménage à Chedabouctou, où les terres sont meilleures. Il commence à cultiver du blé et projette d'installer un moulin à farine et de fabriquer de la bière pour remplacer le vin. Il projette aussi d'extraire des huiles de loups-marins, pour augmenter ses revenus. Efforts désespérés d'un malheureux dont les affaires périclitent.

L'entreprise éprouvait donc de sérieuses difficultés. Sans doute plusieurs dépenses encourues, comme la construction des habitations et le défrichement des terres, pouvaient se considérer comme des placements à longue échéance, qui eussent fini par rapporter. S’il n'y avait eu que des difficultés commerciales, on aurait pu croire que Denys, par son expérience et sa ténacité, aurait fini par les surmonter. Malheureusement, dès le début de l'association, il avait éprouvé des embarras d'autre nature qui aggravèrent la situation.

Nous savons déjà que d'Aulnay avait dépossédé Denys de Miscou et Nepisiguit en 1647. Dès sa mort, Nicolas Denys et son frère, Simon, se rétablirent au Cap-Breton, mais pour subir presque aussitôt de nouvelles traverses des héritiers d'Aulnay, entre autres Emmanuel LeBorgne. Denys possédait sûrement alors des commissions de la Compagnie de la Nouvelle-France. Aucun texte n'en est demeuré, et des historiens en ont mis l'existence en doute. Mais il est invraisemblable qu'il ait pris le risque de construire des habitations, à portée de ses adversaires, sans autorisation. Il mentionne lui-même ces commissions à diverses reprises; quand d'Aulnay s'empara de son fort à Nepisiguit, il nous dit explicitement que ce dernier n'osa, à cause de sa commission, le déposséder tout simplement, mais dressa l'inventaire de ses marchandises et lui en promit le paiement. Plus tard, il nous dit que LeBorgne voulait s'emparer de sa commission. Peut-être les textes entraient-ils en contradiction 
avec l'autorité du gouverneur, ou Denys avait-il négligé de les faire enregistrer ou confirmer par le Roi ? Seules les archives de la Compagnie pourraient éclairer ce point.

En tout cas, sous un prétexte ou un autre, LeBorgne attaqua le fort de Saint-Pierre en 1653 ou 1654 et s'empara de toutes ses marchandises. Par représailles, Denys saisit un navire qui transportait des provisions pour LeBorgne et pratiquait une traite illicite. Il s'empara aussi de pelleteries, à la côte d'Acadie, sur le fils LeBorgne. LeBorgne à son tour faisait saisir les pelleteries de Denys à La Rochelle ... Cet échange d'amabilités pouvait durer longtemps...! ! Dans l'intervalle, Denys avait obtenu une nouvelle commission très explicite confirmée par le Roi, et une nomination comme lieutenant-gouverneur. Afin que personne n'ignore ses privilèges et de ne plus courir le risque d'en perdre les preuves, il les fit imprimer et distribuer. Il avait aussi obtenu en 1655 un arrêt défendant à LeBorgne de le troubler à l'avenir. Mais le règlement financier de leurs différends demeurait pendant. Les pourparlers à ce sujet durèrent trois bonnes années. Deux noms célèbres d'avocats se trouvent mêlés à cette affaire: ceux de Chapelain, le père du poète, et de Chanut, ancien ambassadeur en Suède, ami des Fouquet.

Il semble que Denys avait le bon droit pour lui, mais l'avait un peu outrepassé, et ne put le défendre. S'il déclassait largement LeBorgne en expérience de la pêche et de la traite, il ne pouvait rivaliser avec lui dans l'habitude de la chicane. L'Amirauté de La Rochelle nous montre en effet chaque année le nom de ce marchand impliqué dans de multiples procédures. Sa grande habileté contre Denys fut de compliquer savamment la situation, en l'empêtrant dans trois procès à la fois.

Denys, invoquant ses privilèges, avait fait saisir deux vaisseaux traitants, celui de Marot et un autre appartenant au Basque Martignon. Tous deux lui avaient été adjugés comme de bonne prise, par la sénéchaussée complaisante de Concarneau. Mais, à l'instigation de LeBorgne, le duc de Vendôme intervint et, prenant le parti de Martignon, obtint un arrêt d'emprisonnement contre Denys. Celui-ci, se heurtant à l'influence de Ven- 
dôme, ne put obtenir d'appel et dut se cacher pendant plusieurs mois, pour éviter la prison. Finalement il dut rendre l'un des vaisseaux mais, par une curieuse contradiction, obtint quand même de Vendôme, moyennant $600 \mathrm{~L}$., un arrêt de confiscation pour la forme, qui pourrait servir contre d'autres. Cette affaire nous donne une pauvre opinion de l'intégrité de son Altesse Royale le grand maître de la Marine, le duc de Vendôme.

Pendant ce temps les arbitres réglaient l'affaire DenysLeBorgne aux dépens de Denys. Il n'obtenait aucune compensation pour les pertes subies à la prise du fort Saint-Pierre, et devait au contraire payer 5,000 L. de dédommagement pour les pelleteries saisies sur les LeBorgne. Il était quand même heureux de s'en tirer à ce prix. Car Chalain, lassé de ces chicanes, poussait à un accommodement et songeait même à grouper dans une seule association LeBorgne et Denys. Ce dernier proteste évidemment comme un diable contre cette idée de l'associer à son plus redoutable ennemi. Il accepta donc un peu malgré lui ce règlement, et Fouquet de Chalain, une fois de plus, avança l'argent. Il semble que Denys, en guise de consolation, put garder l'un des navires confisqués, probablement celui des Marot.

Mais ces ruineux procès ne favorisaient guère le commerce. Les acheteurs de fourrures se tenaient sur la réserve, craignant les saisies. Retenu par d'interminables délais, Denys ne put passer en Acadie en 1657 et Prévost, son lieutenant, prit trois mois à traverser. En somme, ces procès coûtèrent sûrement à l'association une perte de 10 à 15,000 L.

A peine tranquille de ce côté, Denys dut entrer en contestations d'un autre genre auprès de la Compagnie, au sujet des limites de sa concession. Ni les Cent-Associés, ni les ministres $\mathrm{du}$ Roi ne prenaient guère la peine de se renseigner sur les vastes domaines qu'ils distribuaient en Nouvelle-France, et l'on a vu souvent de coûteuses complications résulter de leur négligence.

Nicolas Denys, par ses commissions de 1653 et 1654, avait obtenu toute la côte sud du golfe Saint-Laurent, du Cap des, 
Rosiers à Canso, avec les îles adjacentes. Notons tout de suite une première contradiction. L'Ile du Cap-Breton, attribuée explicitement à Denys, avait déjà été concédée aux sieurs Pierre Desportes et Jean Tuffet, en 1636, et appartenait alors au sieur Frotté. La propriété de cette île ne causa heureusement aucune mésentente, puisque Nicolas Denys, en 1664, racheta ces droits pour $2,000 \mathrm{~L}$.

Le nom de Terre-Neuve figure aussi en toutes lettres dans la confirmation royale accordée à Denys en 1654. Ce qui n'empêcha pas le Roi en 1660 d'accorder une autre commission de gouverneur à Isaac de Feuquière et de concéder Plaisance à Nicolas Gargot en 1661. ${ }^{14}$ Comme le gouverneur de Québec exerçait aussi juridiction sur Terre-Neuve, cette île heureuse se trouvait soumise à trois gouverneurs en même temps ! On sait que la Compagnie de Nouvelle-France dressa une forte opposition à Feuquière et Gargot, créatures du surintendant Fouquet. Charles Millon, l'éditeur du Journal de Gargot, a cru pouvoir ranger aussi Denys parmi leurs adversaires. ${ }^{15}$ Mais cette affirmation ne repose que sur des conjectures, que ni les livres ni les lettres de Denys ne justifient. Il s'intéressa toujours davantage à la côte sud du golfe, et s'il forma un jour de vagues prétentions sur Terre-Neuve, il avait de fortes raisons personnelles de ne pas s'opposer aux projets du surintendant Fouquet.

Par contre il protesta vigoureusement contre l'établissement de François Doublet aux Iles-de-la-Madeleine. L'expression «iles adjacentes», insérée dans sa commission, pouvait comprendre l'ile Saint-Jean et les Iles-de-la-Madeleine. Denys du moins l'entendait ainsi, mais ce point manquait de précision. En fait, il ne s'occupa guère lui-même de ces îles, puisque des Basques $\mathrm{y}$ pratiquèrent pendant plusieurs années, avec son autorisation, la chasse aux loups-marins. ${ }^{16}$ Mais quand Doublet en obtint la concession et vint s'y établir avec grand fracas, ce

14 Institut, Coll. Godefroy, Ms 68: 487; capie à la B.N., N.A.F. 9281 (Margry) : 153.

15 C. Millon, Aventures du Rochelais Nicolas Gargot... dit Jambe-deBois (La Rochelle, Ed. Rupella, 1928).

16 D'après le Journal de Doublet et le Mémoire du $\mathrm{Sr}$ de Peyrelongue, Col., C11C, 1:22. 
fut l'occasion de vives altercations. Il est amusant de lire à ce propos les témoignages contradictoires des deux parties. Aux yeux de Denys, Doublet paraît une espèce de hâbleur normand qui, malgré son ignorance, prétend en savoir plus que tous et promet des gains mirifiques. Le journal de Jean Doublet, de son côté, nous représente Denys comme une espèce de matamore batailleur. ${ }^{17}$ Ces deux portraits contiennent probablement chacun au moins une parcelle de vérité.

Denys porta plainte à la compagnie, sans résultat. Il dut endurer les empiètements de Doublet, non sans lui prédire une prompte déconfiture. Ses lettres écrites au lendemain des événements manifestent une indignation dont ses livres, dix ans après, répètent encore l'écho. Il proteste sans doute par mécontentement de voir la compagnie céder facilement à d'autres ce qu'elle lui avait chèrement vendu, mais aussi probablement parce que les Basques lui cédaient, en redevance, une partie de leurs huiles, et qu'il projetait d'entreprendre lui-même bientôt la chasse aux loups-marins.

Une autre concurrence, celle de Canger et de LaGiraudière, lui causa un préjudice incomparablement plus grave, désastreux même. Vers 1659, Denys, sans abandonner Saint-Pierre, s'était établi à la limite sud-est de son domaine, à Chedabouctou, au fond de la baie de Canso. Il y avait amené des engagés et des bestiaux, semé des blés et commencé un établissement considérable. Près de là, à la rivière Sainte-Marie, un Mr de Canger, dont LaGiraudière agissait comme lieutenant, s'était établi depuis 1658. ${ }^{18}$ Denys venait à peine de s'installer que ses voisins obtenaient de nouvelles lettres qui reportaient la limite de leur concession au Cap Saint-Louis, incluant ainsi toute la baie de Canso et le nouvel établissement de Denys. Par ignorance ou mauvaise foi, ils avaient situé le cap Canso au nord de la baie, tandis qu'il se trouve au sud. Cette confusion amena une petite guerre à main armée, qui dura deux ans. LaGiraudière vint

17 Ch. Bréard, Journal du corsaire Jean Doublet de Honfleur (Paris, Perrin, 1887).

18 B.N., N.A.F. 21510:202. Confirmation de concession à M. de Cangé, 8 mai 1668. 
sommer Denys d'évacuer les lieux; celui-ci refusa et se mit en état de défense. L'année suivante un frère de LaGiraudière alla capturer le fort Saint-Pierre. Denys, pour recouvrer SaintPierre, convint de céder plutôt Chedabouctou, et les deux intéressés passèrent en France, soumettre leur différend à la compagnie. Ces messieurs ajustèrent leurs lunettes, consultèrent les cartes, et s'aperçurent avec étonnement qu'ils avaient en effet concédé deux fois le même terrain. Ils annulèrent donc la seconde concession, mais sans aucune réparation pour Denys. Cette erreur lui causait, dit-il, en pertes de salaires, marchandises et vivres gaspillés, bâtiments ruinés, une somme de 53,000 L. et il dut abandonner Chedabouctou. Pour un homme déjà lourdement chargé de dettes, cette nouvelle perte l'enfonçait désespérément. Et l'affaire menaçait de rebondir, puisque Du Canger projetait d'en appeler au Conseil de Marine. Décidément MM. Fouquet et Duquesne devront encore attendre un peu le remboursement de leurs dûs. Ils attendront longtemps.

L'un d'eux, Jacob Duquesne est mort en 1660. Le président Chalain, compromis dans la disgrâce de son cousin en 1661, passa quelque temps à la Bastille. Cela donne à Denys le temps de respirer un peu. Mais Abraham Duquesne, s'il ne semble guère se préoccuper des intérêts de sa belle-sœur, avec qui il a procès, ni de son neveu converti au catholicisme, n'oublie pas ses propres créances et harcelle furieusement Denys. Il le soupçonne de détourner des pelleteries à son profit personnel et envoie au Canada un observateur. Il menace même d'équiper un vaisseau pour venir opérer une rafle, s'emparer de ses établissements et installer un autre agent à sa place. Cristophe Fouquet libéré envoie aussi un homme de confiance faire enquête sur place. Plus généreux, il offre à Denys d'acheter sa concession pour la somme de . . 5,000 L.!

Denys, qui s'est longtemps berçé d'illusions, doit enfin admettre la réalité. Il écrit à Fouquet, le 8 août 1664, une longue lettre désenchantée, la dernière, qui résume assez bien la situation. Il est alors âgé de 66 ans, et ne croit plus possible de tirer aucun profit du Canada. «La pelleterie a diminué du tiers» et, là où il trafiquait seul, il rencontre à présent des concurrents 
acharnés. Il ne croit plus à la pêche sédentaire, car l'hiver canadien impose six mois de saison morte, pendant lesquels il faut nourrir inutilement des hommes et payer des gages. L'agriculture offre peu de ressources; les arbres feraient croire à la fertilité du sol, mais «le fonds n'est rien que roche» et il n'y a pas «deux cent arpents de bonne terre ». Ajoutez à ce tableau les mouches et les maringouins, et vous aurez une pauvre idée $\mathrm{du}$ pays. Denys subit alors une crise de découragement et pousse les choses au noir. Lamentable aveu de défaite, testament d'un banqueroutier.

Il est en effet bien évident à ce moment qu'il ne pourra jamais satisfaire à ses obligations. Duquesne a repris du service dans la Marine; Fouquet intente seul des procédures et obtient un ordre de liquidation. Mais allez donc saisir un créancier qui demeure par delà l'océan, en pleine sauvagerie ! Le seul actif que possède Denys, c'est son domaine, et la Compagnie des Indes, substituée à celle de la Nouvelle-France, vient d'annuler toutes les concessions antérieures. La décision judiciaire ne put donc recevoir d'exécution. Denys, sans désarmer, obtiendra en 1667 une autre commission de la nouvelle Compagnie, et pourra recommencer à neuf.

Dix années s'écoulent. Duquesne, devenu lieutenant général de la Marine, passe la majeure partie de son temps sur les mers. Son épouse, Gabrielle de Bernières, agissant en son nom, signe un accord avec Fouquet de Chalain, afin de poursuivre à frais communs leur créancier. Cette fois encore l'éloignement dut protéger Denys, car en 1684, dans une lettre à Cabart de Villermont, ${ }^{19}$ il demande à ce dernier d'apaiser Duquesne qui réclame toujours. Le débiteur et le créancier moururent tous deux la même année, en 1688, et purent enfin aller régler leurs comptes dans l'autre monde.

Mais ce long épilogue ajoute peu à l'histoire de cette association. En fait, elle se termine en 1664. Denys doit en ce moment à ses associés, en tenant compte des intérêts accumulés, au delà de 100,000 $\mathrm{L}$. Il vit pauvrement à $\mathrm{S}$. Pierre, lui et sa

\footnotetext{
19 Copie à la B.N., N.A.F. 9281 (Margry) : 145.
} 
famille, de ses jardinages et de quelques profits sur la troque des fourrures et du poisson. Tout ce qui lui reste, ce sont quelques fortins de bois rond et son domaine: 200 lieues de côte sauvage. $\mathrm{Au}$ point de vue commercial, l'entreprise se termine par un échec complet.

Mais les marchands perdaient-ils vraiment une somme aussi considérable ? Dès 1658, le passif de 51,520 L. admis par Denys comprenait déjà les intérêts additionnés pendant cinq années. De plus des remises faites par Denys à Jacob Duquesne avant sa mort n'étaient pas déduites: par exemple 10,000 pour le retour d'un vaisseau et 8,000 L. d'une cargaison de poisson en 1658. Dans plusieurs lettres Denys ne cesse de réclamer, outre une diminution d'intérêts, le rajustement de sa dette. Dans une lettre à Fouquet, il prétend même avoir remboursé à peu près tout le capital. Par ailleurs Duquesne et Fouquet avaient réalisé plusieurs profits supplémentaires sur la fourniture des vivres et la revente des fourrures. Dans une occasion entre autres, Fouquet avait gagné $30 \%$ en expédiant des fourrures en Hollande. En tout cas, les marchands ne perdaient que leur argent, tandis que Denys, outre les sommes qu'il avait engouffrées personnellement en bâtisses et en salaires, perdait dix années de labeur et de risques. Il vécut encore vingt ans au Canada, mais cette entreprise avait ruiné son crédit et malgré ses efforts, il ne put s'en relever.

$\mathrm{Au}$ point de vue du peuplement, si les résultats n'atteignirent guère plus de succès, l'échec ne fut pas aussi complet. Des divers postes fondés par Denys, Miscou, Nepisiguit, Saint-Pierre, Chedabouctou, aucun ne survécut immédiatement, mais d'autres en reprirent et continuèrent les défrichements. Des nombreux engagés qu'il fit passer en Acadie, la plupart retournèrent en France. Mais quelques-uns durent demeurer à Port-Royal. De colons stables, il y avait 7 familles en 1662. Plusieurs textes permettent aussi d'attribuer à Denys la venue de missionnaires jésuites au Cap-Breton, en 1658, pour desservir colons et sauvages. ${ }^{20}$ Il laissera aussi son fils Richard et une fille qui épousa LaVallière. Ces résultats représentent sans doute peu de chose, en regard des 80 familles qu'il s'était engagé d'établir par la com- 
mission de 1653. Mais ils suffisent pour que l'aventure de Denys n'ait pas été entièrement perdue.

S'il ne réussit pas mieux, pouvons-nous le blâmer de son échec ? Son aventure, loin d'être exceptionnelle, en représente assez bien une demi-douzaine d'autres, tentées à la même époque. Lui-même en énumère plusieurs: la compagnie de Ryvedon Colbert et Villeneuve, au Cap Sable, se termina par une perte de 80,000 L.; Du Canger en 1664 avait déjà perdu 30,000 L.; la prise de son poste par les Anglais en 1669 acheva de le ruiner; la compagnie de Doublet ne dura que deux ans. A ce martyrologe on peut ajouter le nom d'Aulnay, qui laissait à sa mort une dette de 250,000 L., et celui de LeBorgne, qui mourut aussi ruiné.

Toutes ces entreprises finirent par des échecs plus ou moins retentissants, pour des causes identiques: frais excessifs, rivalités ruineuses, absence d'une politique éclairée, agressions étrangères. La seule façon de fonder des établissements stables, Denys l'a bien vu, consistait à fixer au pays des colons par la pêche sédentaire et la culture du sol. Mais il fallait aussi, pour atteindre ce but, que finisse le règne de la pagaille et de l'incertitude. L'ordre et la sécurité que les compagnies s'avéraient incapables d'établir, seul le Roi pouvait les assurer. C'est la dernière espérance de Denys. "Il n'y a que le Roi, écrit-il en 1664, qui soit capable de le faire valoir, et l'on m'a fait entendre qu'il était en volonté d'avoir tout le pays; je le souhaiterais. » Cet espoir de jours meilleurs empêche Denys de désespérer complètement, et le poussera à publier deux livres pour faire connaître le Canada et suggérer les réformes indispensables.

On n'a souvent voulu voir en Denys qu'un commerçant. Ce marchand, croyons-nous, possédait, quoique de façon un peu fruste, la vision et le caractère d'un colonisateur. Ses idées rejoignent en plusieurs points celles qu'avait formulées Razilly, et que Talon commençait d'appliquer à la même époque au Canada. Il lui a manqué peu de chose pour les réaliser: peut-être plus de prudence, des circonstances favorables, et la chance de vivre vingt ans plus tard, sous la protection d'un Colbert ou d'un Talon. Mais sa vie nous laisse quand même un grand exemple.

20 B.N., Clairambault 1016:493. 
Ses lettres mieux encore que ses livres, nous le montrent au naturel, avec ses qualités et ses défauts : c'est un rude pêcheur, ignorant de l'orthographe et du style, mais doué d'une intelligence incontestable. Endurant et tenace, il recommence toujours, malgré les pires revers. Honnête et loyal, il a grande influence sur les sauvages. Il aime ses enfants, et l'on sent parfois sous sa plume un frisson de sensibilité ou une pointe d'humour. En somme une grande âme, sous une écorce rude. S'il ne réussit que petitement, il nous apparaît cependant comme une grande figure. Il est de ces hommes dont la taille morale domine la mauvaise fortune, et inspire le respect et l'attachement.

Université Saint-Joseph,

René BAUDRY, c.s.c.

Nouveau-Brunswick. 